\title{
Antisense gapmers selectively suppress individual oncogenic p73 splice isoforms and inhibit tumor growth in vivo Stephan Emmrich ${ }^{1}$, Weiwei Wang${ }^{2}$, Katja John1, Wenzhong Li $^{2}$ and Brigitte M Pützer*1
}

\author{
Address: ${ }^{1}$ Department of Vectorology and Experimental Gene Therapy, Biomedical Research Center, University of Rostock, D-18057 Rostock, \\ Germany and ${ }^{2}$ Department of Cardiac Surgery, University of Rostock, D-18057 Rostock, Germany \\ Email: Stephan Emmrich - emmrichs@med.uni-rostock.de; Weiwei Wang - starwxk@googlemail.com; Katja John - katja.john@med.uni- \\ rostock.de; Wenzhong Li - wenzhong@med.uni-rostock.de; Brigitte M Pützer* - brigitte.puetzer@med.uni-rostock.de \\ * Corresponding author
}

Published: II August 2009

Molecular Cancer 2009, 8:6I doi:10.1|186/|476-4598-8-6I

This article is available from: http://www.molecular-cancer.com/content/8/I/6I

(c) 2009 Emmrich et al; licensee BioMed Central Ltd.

This is an Open Access article distributed under the terms of the Creative Commons Attribution License (http://creativecommons.org/licenses/by/2.0), which permits unrestricted use, distribution, and reproduction in any medium, provided the original work is properly cited.
Received: 22 June 2009

Accepted: II August 2009

\begin{abstract}
Background: Differential mRNA splicing and alternative promoter usage of the TP73 gene results in the expression of multiple $\mathrm{NH} 2$-truncated isoforms that act as oncogenes. Abundant levels of these p73 variants in a variety of human cancers correlated with adverse clinical prognosis and response failure to conventional therapies, underscoring their relevance as marker for disease severity and target for cancer intervention. With respect to an equally important role for aminotruncated p73 splice forms $(\Delta T A p 73)$ and $\Delta N p 73$ (summarized as DNp73) in the tumorigenic process, we designed locked nucleic acid (LNA) antisense oligonucleotide (ASO) gapmers against individual species that were complementary to $\Delta \mathrm{Ex} 2$ and $\Delta \mathrm{Ex} 2 / 3$ splice junctions and a region in exon $3 B$ unique for $\Delta N^{\prime}$ and $\Delta N$.
\end{abstract}

Results: Treatment of cancer cells with these ASOs resulted in a strong and specific reduction of tumorigenic p73 transcripts and proteins, importantly, without abolishing the wild-type p73 tumor suppressor form as observed with p73-shRNA. The specific antisense oligonucleotides rescued cells from apoptosis inhibition due to overexpression of their corresponding amino-truncated p73 isoform and decreased tumor cell proliferation. Furthermore, ASO-I I 6 against $\Delta \mathrm{Ex} 2 / 3$ coupled to magnetic nanobead polyethyleneimine (MNB/PEI) carriers significantly inhibited malignant melanoma growth, which correlated with a shift in the balance between endogenous TAp73 and $\Delta \mathrm{Ex} 2 / 3$ towards apoptotic full-length $\mathrm{p} 73$.

Conclusion: Our study demonstrates the successful development of LNA-ASOs that selectively differentiate between the closely related p73 oncoproteins, and provide new tools to further delineate their biological properties in different human malignancies and for therapeutic cancer targeting. 


\section{Background}

The human p73 is a member of the p53 tumor suppressor family, based on substantial structural and functional homologies. Unlike p53, p73 is rarely mutated in human cancers [1]. As an early-recognized feature, the TP73 gene produces multiple transcripts with opposing functions. A detailed analysis of p73 in tumor cells revealed the presence of multiple N-terminally truncated isoforms $(\Delta \mathrm{N}$, $\Delta \mathrm{N}^{\prime}, \Delta \mathrm{Ex} 2, \Delta \mathrm{Ex} 2 / 3$; collectively called DNp73), which lack all or most of the transactivation domain that accounts for the tumor suppressor function of the full-length TAp73 protein. While the $\Delta \mathrm{Np} 73$ transcript is generated from a cryptic promoter in intron 3 , the majority of the aminotruncated p73 variants is produced by alternative exon splicing from the E2F1 responsive promoter in the 5'UTR upstream of the non-coding exon 1. All isoforms fail to induce cell cycle arrest and apoptosis. In addition, they act as dominant-negative competitors for DNA binding and/ or heteroduplex formation with p53 and wild-type TAp73, and confer drug resistance to tumor cells harbouring wild-type p53 and/or TAp73 [2,3]. We also demonstrated that the $\Delta \mathrm{Ex} 2 / 3$ isoform inactivates functions of the retinoblastoma (RB) tumor suppressor protein in cell cycle and differentiation control $[4,5]$. By inactivating both major tumor suppressor pathways in human cells they are functionally analogous to several viral oncoproteins. In this sense, overexpression of $\Delta \mathrm{Ex} 2 / 3$ and $\Delta \mathrm{N}$ has been shown to transform fibroblasts to tumorigenicity in nude mice $[6,7]$, and drive carcinogenesis in transgenic mice in vivo [8].

NH2-truncated p73 forms were found frequently elevated in various types of tumor cells and primary malignancies from patients, but not in the surrounding normal tissues [9]. Beyond, increased DNp73 expression levels are strongly associated with a worse recurrence-free and overall survival of patients, and poor prognosis features such as lymph node metastasis and vascular invasion [10]. Specifically, overexpression of $\Delta \mathrm{Ex} 2 / 3 \mathrm{p} 73$ and $\Delta \mathrm{Np} 73$ was associated with advanced pathologic tumor stages. Likewise, alterations in the relative levels of TAp73 and the apparently tumor-specific aberrant expression of individual oncogenic p73 species, that might account for a shift in the net function of p73 from proapoptotic to prosurvival, have been shown to correlate with prognosis in some cancers. All this strongly underscores that the expression pattern of $\mathrm{NH} 2$-truncated $\mathrm{p} 73$ is an important determinant of tumor development and the cellular response to treatments, making it a biological relevant target for cancer prevention and therapy.

Although there is increasing evidence for an equally important role of individual amino-terminal p73 splice variants and $\Delta \mathrm{Np} 73$ in the tumorigenic process, we are currently unable to evaluate their oncogenic potency under physiological conditions in vivo. The aim of our study was to develop mono-specific inhibitors for the majority of NH2-truncated alternative-spliced p73 isoforms that allow differential knockdown of all closely related oncoproteins in growing human tumors.

\section{Methods \\ Cell Culture, Viruses and Fluorescence Microscopy}

Human H1299 lung cancer cells, human embryonic kidney HEK 293 cells, and human WI-38 lung fibroblasts were maintained in Dulbecco's Modified Eagle Medium (DMEM; Invitrogen, Karlsruhe, Germany) supplemented with $10 \%$ fetal calf serum (Biochrom, Berlin, Germany). Human SK-Mel-29 melanoma cells were cultured in DMEM containing sodium pyruvate. Medium contained $2 \mathrm{mM}$ L-glutamine, $100 \mu \mathrm{g} / \mathrm{ml}$ penicillin, $100 \mathrm{U} / \mathrm{ml}$ streptomycin, and 1,25 $\mu \mathrm{g} / \mathrm{ml}$ amphotericin B. The adenoviral (Ad) vectors Ad-shGFP and Ad-shp73 have been described elsewhere [11]. Adenovirus infection was carried out at a multiplicity of infection (MOI) that allows $100 \%$ transduction of target cells. FITC and GFP imaging was performed by fluorescence microscopy at indicated time points.

\section{Antisense Oligonucleotides, shRNA and Transient Transfections}

Oligonucleotide (ON) LNA modifications and FITC-labeling was conducted by BioTeZ GmbH. ASO sequences were the following: ASO-115 5'-CTGTCTGGTTCCCTGCAGCC-3', ASO-116 5'-GATTGAACTGGGCCTGCAGC3', and ASO-185/451 5'-GGAACTGGTGTCCCGTGGGA3' (LNA bases are bold). The non-sense control ON (nsc) with 5'-TAACCGTTTCTTCCTCGTCC-3' serving as a negative control was verified by NCBI blastn search. For in vivo experiments a scrambled ON (sc) to ASO-116 with the same base content was generated using the siRNA Wiz$\operatorname{ard}^{\mathrm{TM}}$ platform at http://www.sirnawizard.com (ASO-sc: 5'-GCCCAGAAGGTGCCTAGGTT-3'). ASO transfections were performed with Lipofectamine 2000 (Invitrogen, Karlsruhe, Germany). Cotransfection experiments were carried out with $2 \mu \mathrm{g}$ plasmid mixed with respective amounts of antisense molecules. Plasmid encoding shRNA was transfected using Effectene (Qiagen, Hilden, Germany). The RNA interference target sequence was 5'GGCCATGCCTGTTTACAAG-3'.

\section{Quantitative Real-Time PCR (qPCR)}

Total RNA was extracted with the NucleoSpin Kit (Macherey-Nagel, Düren, Germany) and reverse transcribed with Omniscript RT (Qiagen, Hilden, Germany) using Oligo $(\mathrm{dT})_{18}$ primer. The cDNA sample was mixed with iQ SYBR Green Supermix (Biorad, München, Germany). QRT-PCR was performed on iQ5 Multicolor Real-Time PCR Detection System (Biorad, München, Germany) using 1/10 volume of RT reaction. Relative gene expres- 
sion was calculated using iQ5 Optical System Software. Primer sequences are available upon request.

\section{Western Blotting}

Cells were lysed in RIPA buffer (50 mM Tris-HCl (pH 7.2), $150 \mathrm{mM} \mathrm{NaCl}, 1 \%$ sodium deoxycholate, $0.1 \%$ SDS, $0.1 \%$ Triton X-100, $5 \mathrm{mM}$ EDTA) supplemented with protease inhibitor mixture Complete Mini (Roche Applied Science, Mannheim, Germany) and total protein concentration was quantified by a modified Bradford assay. Equal amounts of protein were separated by SDS-PAGE, transferred to nitrocellulose membranes, and probed with anti-p73 ER-15 (BD Biosciences, Heidelberg, Germany), anti- $\Delta$ Np73 (Imgenex, San Diego, CA, USA) or anti- $\beta$ Actin (Sigma-Aldrich, Taufkirchen, Germany). Primary antibodies were detected with appropriate secondary antibody-horseradish peroxidase conjugates according to the enhanced chemoluminescence protocol.

\section{Hoechst 33342 Staining and Caspase-3 Activity}

After treatment, cells were incubated with Hoechst 33342 at $1 \mu \mathrm{g} / \mathrm{ml}$ for $15 \mathrm{~min}$ and subjected to fluorescence microscopy. Hoechst-stained cells were harvested, counterstained with $10 \%$ trypan blue solution, and counted in a hämcytometer. Caspase-3 activity was assayed using the ApoAlert kit (Takara Bio, Saint-Germain-en-Laye, France) as described by the manufacturer. Absorbance was measured at $405 \mathrm{~nm}$ in a spectrophotometer.

\section{Cell Proliferation Assay}

Cells were seeded at a density of $5 \times 10^{4}$, serum-starved for $24 \mathrm{~h}$, and transfected with PEI-ASO complexes at day 1 and 3 after plating. DNA synthesis was measured by Cell Proliferation BrdU (5-bromo-2'-deoxyuridine)-ELISA (Roche Applied Science, Mannheim, Germany) according to the instructions.

\section{Magnetic Nanobead (MNB)/Polyethyleneimine (PEI)/DNA Complex Formation}

Branched PEI (average MW 25 000) (Sigma-Aldrich, Taufkirchen, Germany) was purified through dialysis in three changes of a 100-fold volume excess of water, lyophilized and re-hydrated before use. PEI/ASO complexes were prepared at the N/P ratio of 8 . The PEI solution and DNA solution at a given $\mathrm{N} / \mathrm{P}$ ratio were diluted in $5 \%$ glucose to ensure iso-osmolarity for transfection experiments and in vivo application. PEI was added to DNA and immediately mixed and incubated for $30 \mathrm{~min}$ at RT. For intratumoral injections, PEI/ASO complexes were conjugated with streptavidin-coated magnetic nanobeads (MNBs) (Promega, Mannheim, Germany) by vortexing for $30 \mathrm{~s}$ followed by incubation at RT for $30 \mathrm{~min}$. The resulting MNB/polymer/ASO complexes are stable in aqueous solution and can be stored at $4{ }^{\circ} \mathrm{C}$ for several days.

\section{Animal Experiments and In Vivo Imaging}

Tumors were established by subcutaneous (s.c.) injection of $10^{7}$ SK-Mel-29 cells into the rear flanks of 6 - to 8-weekold female athymic nu/nu mice. Animals with palpable tumors were randomized in three groups to ensure uniform distribution: Group A untreated control $(\mathrm{n}=9$ tumors), group B treated with PEI/ASO-sc ( $\mathrm{n}=8$ tumors), and group $\mathrm{C}$ treated with PEI/ASO-116 ( $\mathrm{n}=13$ tumors). For magnetic force transduction, animals with tumors of diameter $>8 \mathrm{~mm}$ were randomized into two groups (group D: MNB/PEI/ASO-sc, $\mathrm{n}=6$ tumors; group E: MNB/ $\mathrm{PEI} / \mathrm{ASO}-116, \mathrm{n}=10$ ). Mice were anaesthetized by intraperitoneal injection of body-weight adapted doses of $10 \%$ ketamine and 2\% xylazin. Oligonucleotide complexes were intratumorally administered at $1.5 \mathrm{mg} / \mathrm{kg} /$ day. In magnetic bead guided experiments, a magnet sized $4 \times 1$ $\mathrm{mm}$ with 1.41-1.45 $\mathrm{T}$ was placed closely adjacent to the tumor. Tumor volumes were measured with calipers and calculated from the longest diameter and average width by assuming a prolate spheroid shape (tumor volume $=\pi$ /

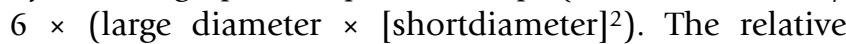
tumor volume (RTV) was determined using $R T V=V_{i} / V_{0}$, where $V_{i}$ is the daily-measured tumor volume and $V_{0}$ is the initial tumor volume. After treatment, mice were sacrificed and representative tumor specimen of each experimental group were dissected. Total RNA was extracted and quantities of $\Delta \mathrm{Ex} 2 / 3 \mathrm{p} 73$ and TAp73 transcripts were determined by qPCR. All animal procedures were conducted in adherenceto ethical standards and with approval of the local Animal CareCommittee.

For fluorescence detection, PEI was labeled by Oregon green 488 Protein labeling kit (Invitrogen, Karlsruhe, Germany) and injected intratumorally into mice. In vivo imaging was performed at 1,8 and $24 \mathrm{~h}$ after injection. Images were acquired using NightOwl LB981 imaging system (Berthold Technologies, Bad Wildbad, Germany) with an exposure time of $100 \mathrm{~s}$. For colocalization of the fluorescent image on the animal body, gray scale and pseudocolor images were merged. Quantification of signal intensity in all animals was performed by WinLight 32 Software.

\section{Computational Analysis and Secondary Stucture Predictions}

Human genomic sequences for the 5'UTRs of TP73 transcripts were obtained from ENSEMBL database. The binding energies as well as the propability matrices for singlestranded target sequences were calculated using the Sfold server application module Soligo. Each N-terminal p73 transcript was calculated with the C-terminal configurations $\alpha, \beta, \gamma$ and $\delta$, and their propability diagrams were merged at the indicated nucleotide positions. In silico secondary structure prediction was carried out using the CLC RNA Workbench Software (CLC Bio, Aarhus N, Den- 
mark). For each $\mathrm{N}$-terminal p73 transcript C-terminal configurations $\alpha, \beta, \gamma, \delta$ and $\varepsilon$ were used to calculate the mRNA structure. The respective ASO epitopes at the N-termini of these mRNAs were then compared for structural identity in the ASO target sequence.

\section{Statistical Analysis}

Statistical analysis of tumor growth in nude mice was performed with one-way analysis of variance (ANOVA). Variances in BrdU incorporation, caspase activation and viable cell counts were compared with an unpaired Student's t-test (two-sided).

\section{Results and Discussion \\ Design of LNA-DNA gapmers against oncogenic NH2- truncated $p 73$ isoforms}

Differential mRNA splicing and alternative promoter usage of the human TP73 gene results in the expression of multiple N-terminally truncated forms $(\Delta \mathrm{Ex} 2, \Delta \mathrm{Ex} 2 / 3$, $\Delta \mathrm{N}^{\prime}, \Delta \mathrm{N}$; Figure $1 \mathrm{~A}$ ) that act as oncogenes. Current evidence suggests an equally important role for individual p73 splice variants and $\Delta$ Np73 in the tumorigenic process, but there is no attempt so far for their selective silencing without concomitant reduction of the apoptotic TAp73 form. In order to develop mono-specific inhibitors for each amino-terminal isoform, we used a modified antisense technology. According to previous reports indicating that ONs with locked nucleic acids (LNA) at their 3'and 5 '-ends efficiently recruit RNase $\mathrm{H}$ and promote degradation of target RNA $[12,13]$, we designed 20-mer LNADNA gapmers with a central DNA region of 12 nucleotides and 3'-5' ends of four LNA monomers. LNA bases have been shown to confer resistance to nucleases when incorporated at the 5 ' and 3 ' ends of oligomers [14]. Antisense specificity for $\Delta \mathrm{Ex} 2 \mathrm{p} 73$ and $\Delta \mathrm{Ex} 2 / 3 \mathrm{p} 73$ was achieved by using the splice junction of each exon deletion variant (Figure 1B). The ASO targeting $\Delta \mathrm{N}^{\prime}$ and $\Delta \mathrm{N}$ was directed against a region in the intron-derived exon $3 \mathrm{~B}$, which is unique to both isoforms.

The optimal target sequence was determined in silico by three parameters: (a) a probability profile for singlestranded regions in the mRNA, which serve as docking stations for initial oligo annealing $[15,16]$, (b) a minimum free energy model of the secondary structure of the mRNA with a more recent compilation of Turner's free energy parameters [17], and (c) the binding energies for a given ASO sequence on the mRNA [18]. The probability plot for $\triangle \mathrm{Ex} 2 \mathrm{p} 73 \mathrm{mRNAs}$ indicates single-strands downstream of nucleotide (nt) position 120 and around position 130 [see Additional file 1A]. This was confirmed by secondary structure prediction for $\Delta \mathrm{Ex} 2 \mathrm{p} 73$ transcripts, where two bulges of more than 4 bases were present at positions 121 and 130, exposing the splice junction for oligo binding [see Additional file 1E]. Since mRNA 3D-structure depends predominantly on nearest neighbour interactions, alterations in the C-terminus may affect folding of $\mathrm{N}$-terminal domains [19]. Therefore, structure predictions were carried out for several C-terminal isoform configurations. Binding energies for ASO-115 (number indicates starting base of ASO position on the mRNA) targeting $\triangle$ Ex2p73 mRNA reached values around $-8 \mathrm{kcal} / \mathrm{mol}$ [see Additional file 2]. According to the Soligo algorithm using $\leq-8 \mathrm{kcal} / \mathrm{mol}$ as default filter criteria for potent ASOs, the values for ASO-115 were considered sufficient. Similar results were obtained for ASO-116. Analysis for singlestranded sections revealed a high probability for unpaired mRNA at the splice junction of $\Delta \mathrm{Ex} 2 / 3 \mathrm{p} 73$ transcripts, which was confirmed by the predicted structure model [see Additional file $1 \mathrm{~B}, \mathrm{~F}$ ]. In the $\Delta \mathrm{Np} 73$ and $\Delta \mathrm{N}^{\prime} \mathrm{p} 73 \mathrm{ASO}$, where the target region is of different location in each transcript owing to distinct transcriptional and mRNA maturation processes, ASO-185/451 denotes the starting positions for the ON in both isoforms. The probability plots and secondary structures of $\Delta \mathrm{Np} 73$ showed the minimum of 4 unpaired bases, which are sufficient for initial oligo annealing [see Additional file 1C, G] [19]. Likewise the predicted epitopes and probability plots for $\Delta \mathrm{N}^{\prime} \mathrm{p} 73$ transcripts suggest mRNA accessibility at the target region of ASO-185/451 [see Additional file 1D, H].

\section{ASOs induce selective suppression of target RNAs}

To ensure efficient internalization of the ASOs into target cells, we determined the amount of oligonucleotide uptake after 8,16 and $24 \mathrm{~h}$ of transfection using a concentration range between 100-250 nM fluorescein isothiocyanate (FITC) labeled ASO. All LNA-DNA gapmers showed a similar high transfection efficiency in HEK293, H1299, and SK-Mel-29 cells overexpressing individual DNp73 isoforms, and similar intracellular distribution patterns with clear nuclear, perinuclear, and diffuse cytosolic staining (data not shown). The capacity and specificity of the designed antisense gapmers to reduce endogenous levels of $\Delta \mathrm{Ex} 2, \Delta \mathrm{Ex} 2 / 3, \Delta \mathrm{N}^{\prime}$, and $\Delta \mathrm{N}$ transcripts was subsequently analyzed by qRT-PCR in HEK293 containing all $\mathrm{N}$-terminally truncated isoforms at higher levels (Figure 2A). Based on previous results indicating that RNase $\mathrm{H}$ enzymatic activity is stimulated as early as $1 \mathrm{~h}$ after ASO treatment [20], antisense molecules were transfected at a concentration of $250 \mathrm{nM}$ into HEK293 cells and gene silencing efficiencies have been monitored over 2, 4, and $6 \mathrm{~h}$ after treatment. The effect of a non-sense control ON is shown for comparison. In these cells, ASO-115, 116, and $185 / 451$ directed against the splice forms $\Delta \operatorname{Ex} 2, \Delta \operatorname{Ex} 2 /$ 3 or $\Delta \mathrm{N}^{\prime}$ induced the strongest antisense effect after $6 \mathrm{~h}$ compared to the nsc-gapmer. At this time point, we measured a 35- to 45-fold reduction of gene expression for both ASO-115 (Figure 2B) and ASO-116 (Figure 2C), whereas ASO-185/451 promoted a weaker (5-fold) but still significant suppression of the $\Delta \mathrm{N}^{\prime}$ mRNA (Figure 2D). 
A

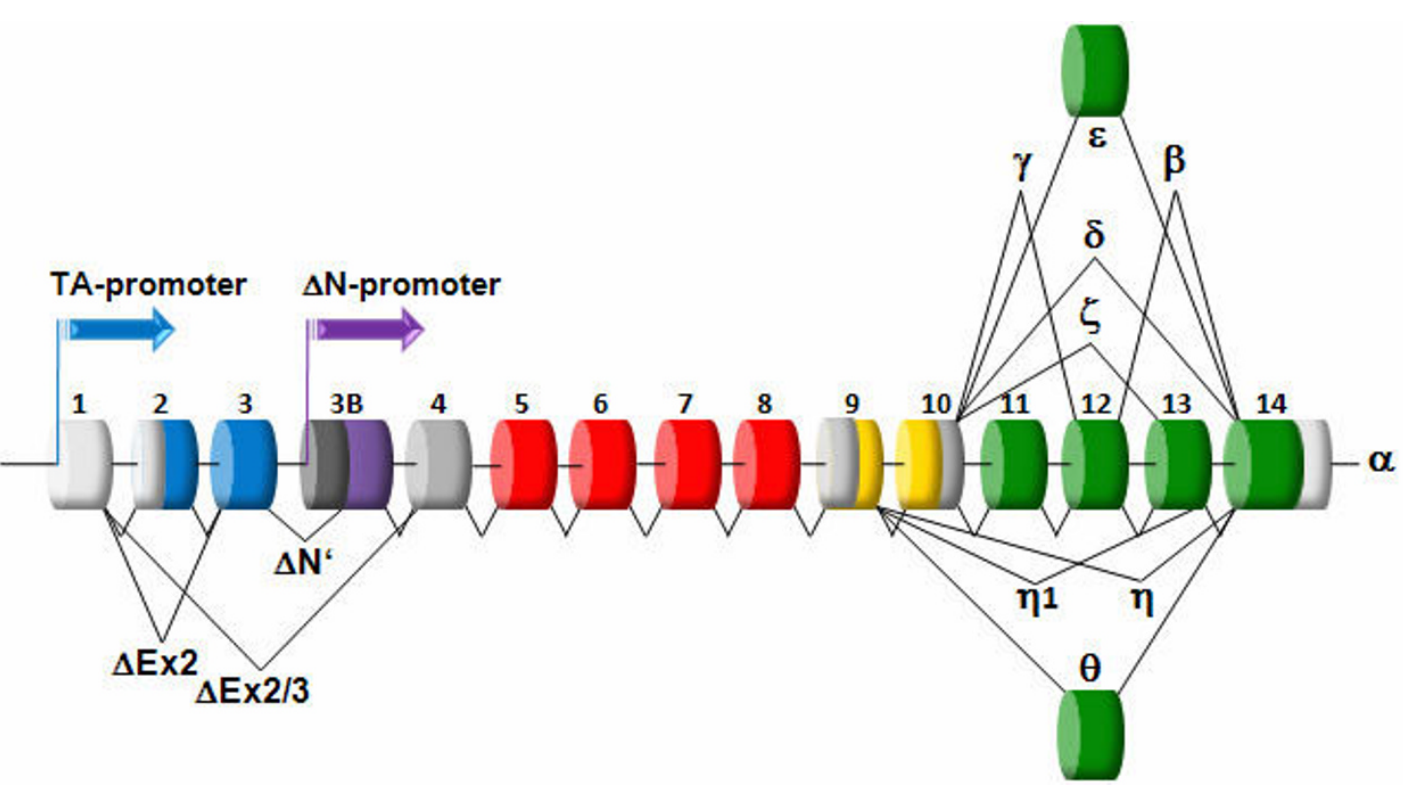

$\mathrm{B}$
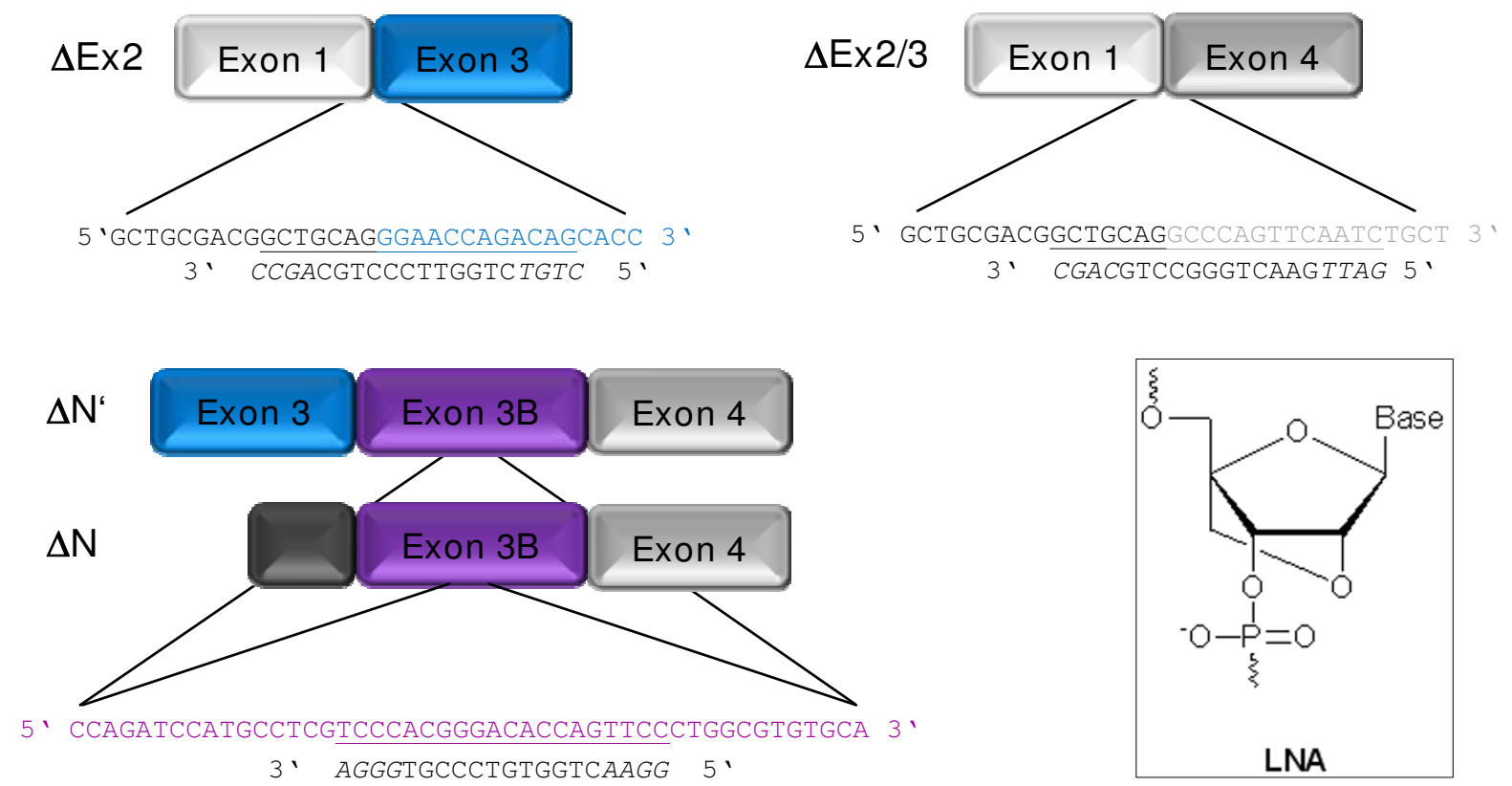

\section{Figure I}

Site-directed targeting of NH2-truncated p73 mRNAs by LNA gapmers. (A) Structure of the human TP73 gene. Exons are shown as boxes and structured according to the domain: transactivation domain (blue); exon 3B-derived coding sequence (purple); DNA-binding domain (red); oligomerization domain (yellow); $\mathrm{COOH}$ terminus (green). C-terminal splice variations are indicated. The transcriptional start sites of the two promoter regions (TA-promoter, $\Delta \mathrm{N}$-promoter) are marked by arrows. Aberrantly spliced transcripts regulated by the TA-promoter are labeled $\Delta \mathrm{E} \times 2, \Delta \mathrm{E} \times 2 / 3$, and $\Delta \mathrm{N}^{\prime}$. The unique $5^{\prime}$ untranslated region of the $\triangle \mathrm{N}$ transcript is colored grey. (B) Binding sites of gapmer ASOs in different DNp73 transcripts. ASO-I I5/ Ex2p73, ASO-I I6/ Ex 2/3, and ASO-185/45I directed against $\Delta N^{\prime} p 73$ and $\Delta N p 73$ mRNA. LNA bases are in bold. 


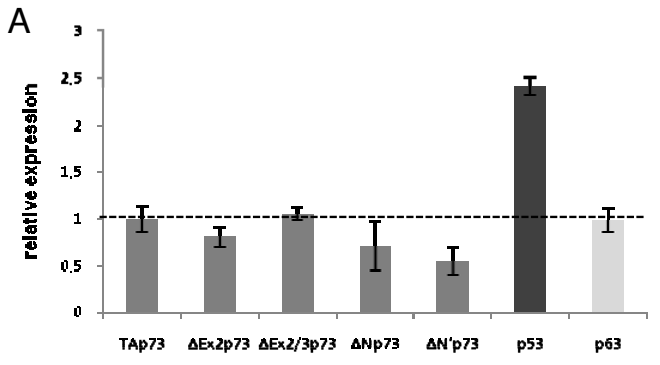

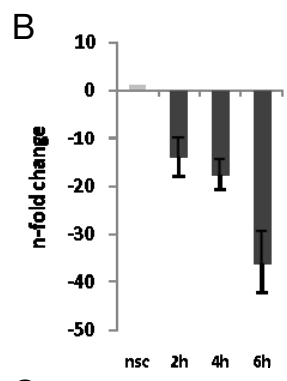

C

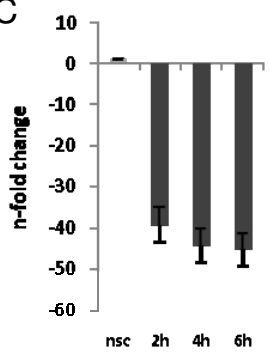

D
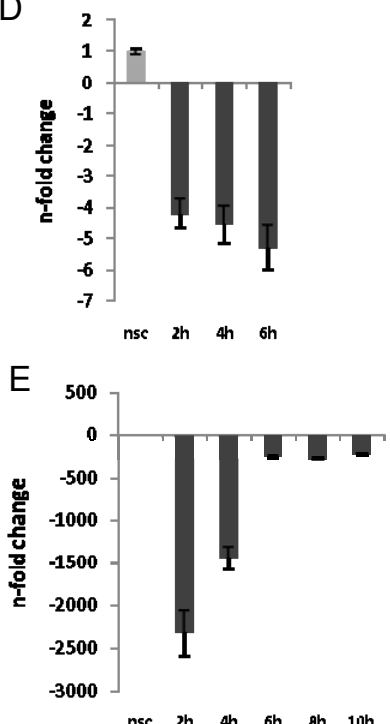

$\mathrm{F}$

nsc $\square 115$

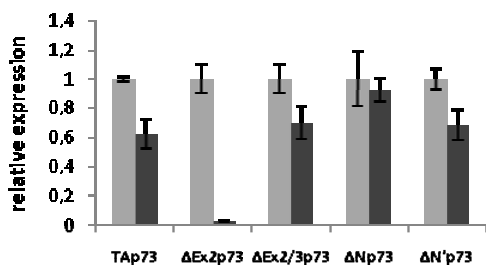

G

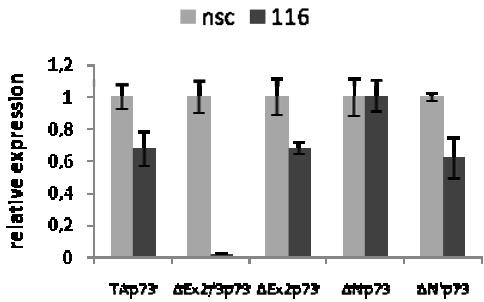

$\mathrm{H}$

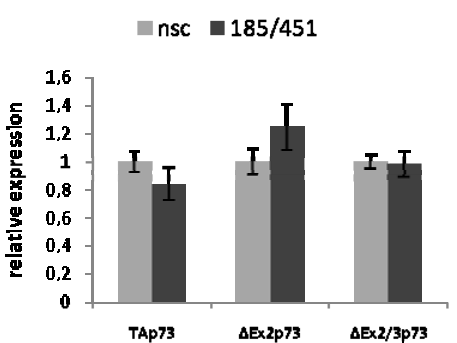

Figure 2

Efficacy and specificity of ASOs for endogenously upregulated p73 transcripts. (A) Quantitative RT-PCR of endogenous expression of TAp73 and amino-truncated p73 transcripts relative to p53 and p63 in HEK293 cells. The broken line indicates the baseline level of DNp73 transcripts. Cells were transfected with $250 \mathrm{nM}$ of ASO-I I5 (B, F), ASO-I I6 (C, G), and ASO-185/45I (D, E, and H). Antisense effects on transcript levels were quantitated by real-time PCR at indicated time points after treatment. Fold expression was calculated after normalization with RPS9 relative to non-specific control-ASO (nsc). Averages and standard deviations of at least three independent experiments are shown. 
Quantitative analysis of the silencing efficiency of the ASO-185/451 antisense gapmer against its second target transcript, revealed a substantially higher inhibitory effect with a more than 2000-fold reduction of the $\Delta \mathrm{N}$ mRNA level already $2 \mathrm{~h}$ following treatment relative to the nscASO (Figure 2E). Repeated experiments provided similar results with rendering the $\Delta \mathrm{Np} 73$ transcript barely detectable during the first $4 \mathrm{~h}$ post transfection. The following time points showed a constant 225- and 279-fold suppression, suggesting a prolonged duration of gene silencing upon amplified initial repression intensity.

The main goal of our study was to design ASOs that allow specific knockdown of individual oncogenic p73 forms. As demonstrated in Figure 2F, ASO-115 induced a complete decrease of $\Delta \mathrm{Ex} 2 \mathrm{p} 73 \mathrm{mRNA}$ to $0.03 \%$ of the control, whereas expression of TAp73 and other non-targeted p73 variants remained largely unaffected. Similar data was obtained with the ASO-116 against $\Delta \mathrm{Ex} 2 / 3 \mathrm{p} 73$ (Figure $2 \mathrm{G}$ ). Interestingly, both ASOs targeting the splice junction of exon 1 have very little effect on $\Delta \mathrm{Np} 73$ transcripts, which lack exons 1 to 3 due to alternative promoter usage. Analogous to this, ASO-185/451 revealed no silencing impact on the $\Delta \operatorname{Ex} 2$ and $\Delta \mathrm{Ex} 2 / 3$ transcripts because of the absence of exon $3 \mathrm{~B}$ in these variants (Figure $2 \mathrm{H}$ ). These results indicate that all developed ASOs exhibit a selectively high inhibitory efficiency against their target, resulting in no or low knockdown of other p73 isoforms. Comparable high isoform-specific knockdown activities of these ASOs were also observed in H1299 tumor cells with amino-truncated p73 transcripts upregulated [see Additional file 3].

\section{Effect of site-directed targeting on DNp73 protein level}

As endogenous levels of all amino-truncated $\mathrm{p} 73$ proteins are hardly detectable in a single cell line, expression plasmids containing the transcript-specific 5'UTRs for the distinct isoforms were cotransfected with $500 \mathrm{nM}$ of the antisense molecules into H1299 cells and analyzed by Western blot. Due to the inherently high stability of the oncogenic proteins, we measured their level in specific LNA gapmer- and control ON-treated cells after $24 \mathrm{~h}$. The levels of full-length TAp73 and DNp73 forms in H1299 cells treated with LNA-DNA are shown in histograms. Following a single transfection, ASO-115 strongly reduced the level of $\triangle \operatorname{Ex} 2 \mathrm{p} 73$ protein up to $30 \%$ of control, but not TAp73 (Figure 3A). A nearly complete disruption of the $\Delta \mathrm{Ex} 2 / 3 \mathrm{p} 73 \alpha$ protein to less than $5 \%$ of the nsc-oligonucleotide was achieved by the treatment with ASO-116. ASO-185/451 but not the negative nsc-ASO significantly suppressed both targets on protein level, although protein derived from the $\Delta \mathrm{Np} 73 \alpha$ plasmid was reduced more efficiently than $\Delta \mathrm{N}^{\prime}$. These findings correlate with the obtained qPCR results, where ASO-185/451 targeting $\Delta \mathrm{Np} 73$ and ASO-116 directed against $\Delta \mathrm{Ex} 2 / 3$ showed the strongest suppression activity. In addition, we cotransfected the two different amino-terminal isoforms $\Delta \mathrm{Ex} 2 / 3$ and $\Delta \mathrm{Np} 73$ along with one of the antisense molecules. As demonstrated in Figure 3B, ASO-116 and vice versa ASO$185 / 451$ inhibited their target in a highly specific manner, while protein expression of the second $\Delta \mathrm{Np} 73$ or $\Delta \mathrm{Ex} 2 /$ 3 p73 isoform in each case remained unchanged. Importantly, consistent with the PCR data, none of the designed LNA gapmers against the oncogenic variants of $\mathrm{p} 73$ has an obvious effect on the amount of TAp73 protein (Figure 3A).

This is in sharp contrast to the effect of a p73-shRNA. The use of RNAi is restricted to sequences that fullfill special criteria, such as the GC content, A at position 19 or absence of internal repeats [21]. According to these parameters, the target sequence of the p73-shRNA is located in exon 5 of the transcript. In contrast to the data observed with the NH2-truncated isoform-specific gapmers, transfection of HEK293 cells with a plasmid encoding shRNA against p73 resulted in a general silencing of TAp73 and oncogenic forms up to $80 \%$ of the scrambledshRNA, with the strongest potency against the wildtype transcript (Figure 3C, upper panel). This unspecific inhibitory effect was also confirmed on protein level. In H1299 cells infected with an adenoviral vector expressing p73shRNA, we found all N-terminal isoforms and TAp73 equally suppressed at $48 \mathrm{~h}$ after infection (Figure 3C, bottom panel) compared to the control virus Ad-shGFP. From these results, we conclude that site-directed targeting of $\Delta \mathrm{Ex} 2$ and $\Delta \mathrm{Ex} 2 / 3$ splice junctions and a unique region in Exon $3 \mathrm{~B}$ for $\Delta \mathrm{N}^{\prime}$ and $\Delta \mathrm{N}$ by antisense $\mathrm{ON}$ is a feasible technique for selective knockdown of individual p73 oncogenes.

\section{Antitumorigenic activity of DNp73 ASOs}

Previous studies have shown that NH2-truncated p73 species inhibit apoptosis by p53 and TAp73 in cancer cells $[3,7,22]$. Consequently, the proapoptotic function of these proteins could be restored by depleting the pool of antagonistic p73 proteins. After having shown that the designed ASOs specifically silence DNp73 forms, we determined whether they also influence cellular survival. Figure $4 \mathrm{~A}$ shows that $\Delta \mathrm{Ex} 2 / 3 \mathrm{p} 73 \alpha$ and $\Delta \mathrm{Np} 73 \alpha$ overexpression markedly reduced the response of normal fibroblasts to genotoxic damage induced by doxorubicin compared to pcDNA3.1 + ASO-nsc treated cells with clear apoptotic features. In contrast, the apoptosis antagonizing effect of both oncogenic p73 forms significantly declined after cotransfection of their specific ASO resulting in increased cell killing over time. Accordingly, while the DNp73 isoforms partially rescued WI-38 cells from DNA damage, isoform-specific ASOs substantially enhanced caspase 3 activity (Figure 4B). 
A

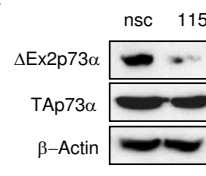

$\square$ nsc $\square 115$

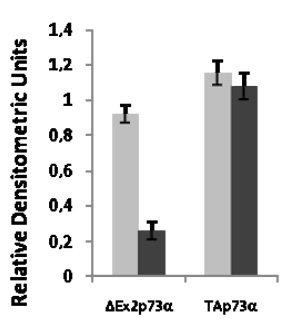

B
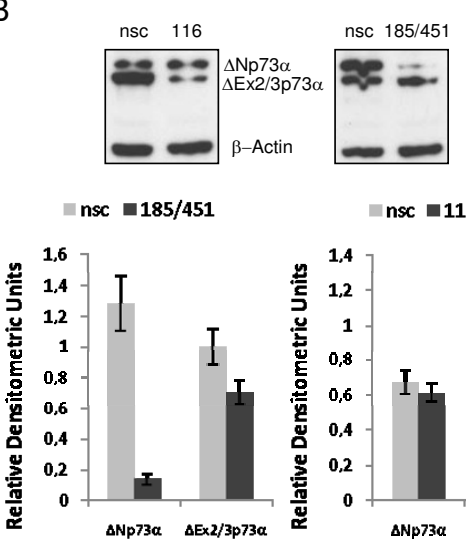

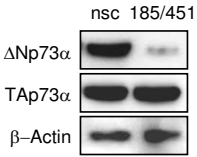

$\| \mathrm{nsc} \backsim 116$

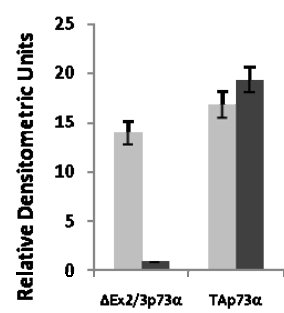

nsc $\square \mathbf{1 1 6}$

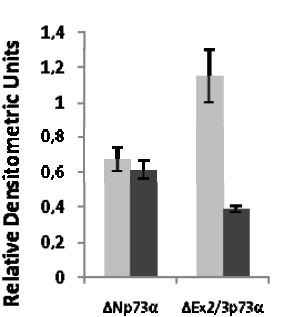

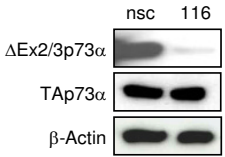

nsc $\square 185 / 451$

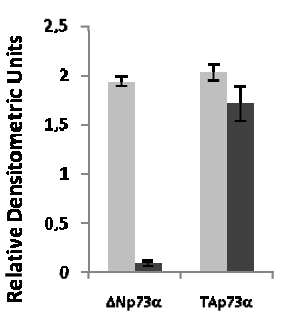

C

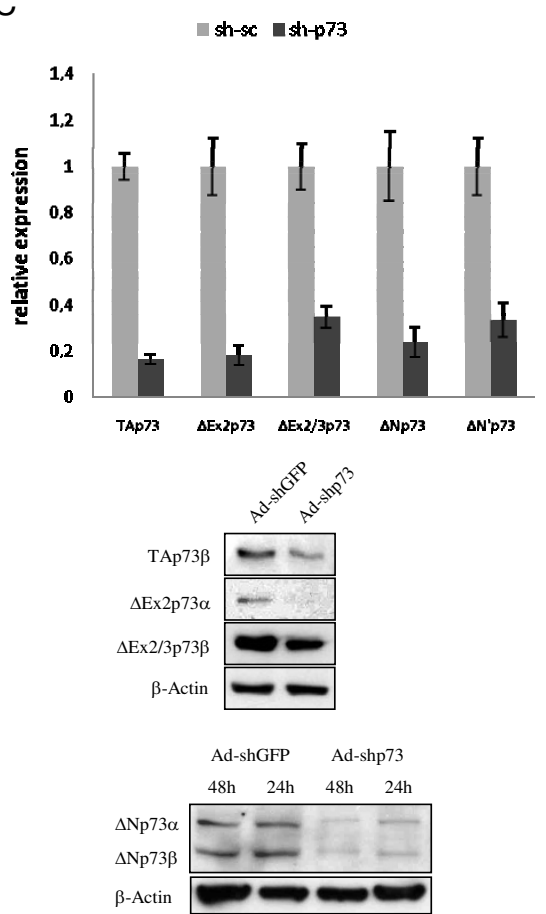

Figure 3

Knockdown effect of NH2-isofom specific ASOs on protein level compared to p73-shRNA. (A) Western blot analysis showing the levels of full-length TAp73 and amino-truncated p73 forms $24 \mathrm{~h}$ following cotransfection of $2 \mu \mathrm{g} p 73$ expression plasmids and $500 \mathrm{nM}$ antisense or nsc LNA-DNA gapmers. Actin was used as a loading control. Protein bands were quantitated in relative software units by the Bio-Imaging-Analyzer (Fuji) using the TINA program (shown as fold induction or reduction, respectively, normalized to actin control bands. (B) Protein levels of $\Delta \mathrm{E} \times 2 / 3$ and $\Delta \mathrm{Np} 73$ in $\mathrm{HI} 299$ cells cotransfected with $\Delta \mathrm{Ex} 2 / 3 p 73$ and $\Delta \mathrm{Np} 73$ expression plasmids along with ASO-II6 (left) or ASO-185/45I (right) compared to control-ASO. Relative densitometric units analyzed as described in $A$ are shown in the bottom panel. (C) QPCR indicating the endogenous expression levels of p73 isoform mRNAs normalized to RPS9 in HEK293 cells at $48 \mathrm{~h}$ after transfection with I $\mu \mathrm{g}$ p73-shRNA encoding plasmid relative to levels in control sh-sc treated cells (set as I). Bar graphs show results from three independent experiments. Data are the mean \pm SD. Immunoblot of HI 299 cells with endogenous $\Delta \mathrm{N}$ levels and transfected with I $\mu \mathrm{g}$ of expression plasmids for TAp73, $\Delta \mathrm{E} \times 2$, and $\Delta \mathrm{E} \times 2 / 3$. Cells were treated with Ad-shp73 or Ad-shGFP at moi 20. Forty-eight hours after infection, cells were lysed, and extracts were probed with anti-p73 (ER-15) antibody. $\beta$-actin was used for equal loading (bottom panel). 
Finally, in an effort to corroborate the role of single amino-truncated p73 species in the tumorigenic process, we tested DNp73-specific ASOs in tumor xenografts established from SK-Mel-29 melanoma cells. These rapidly growing tumors, exhibiting major defects in p53 related apoptosis pathways [23], were selected for our studies due to their high level expression of endogenous $\Delta \mathrm{Ex} 2 / 3$ (Figure $5 \mathrm{~A}$ ), which has so far been implicated in the induction of tumor growth when overexpressed $[7,8]$. For efficient delivery of antisense molecules, ASO-116 was complexed to synthetic nanopolymer PEI [24]. Analysis of the PEI/ ASO-116 polyplexes in cultured SK-Mel-29 cells showed a strong 6-to 9-fold decrease of target mRNA level after transfection (Figure $5 \mathrm{~B}$ ). This inhibitory effect on $\Delta \mathrm{Ex} 2 /$ 3 p73 expression was associated with a significantly reduced proliferation rate by PEI-ASO-116 over 5 days compared to untreated or PEI/ASO-sc treated cells as demonstrated by BrdU-ELISA (Figure 5C). Our data confirm that p73 species-specific ASOs conjugated to PEI are biologically active. Using fluorescence-labeled PEI/ASO-116, signal intensity peaked at $1 \mathrm{~h}$ after intratumoral injection, indicating ASO distribution within the whole tumor (Figure 5D). Although the complex decreased over time, a stabil fraction remained detectable in the tumor after $24 \mathrm{~h}$, which is sufficient to ensure continuous availability of ASO over the treatment period at daily administration.

In order to improve in vivo administration, magnetic nanobeads (MNBs) were used to prevent diffusion of the coupled PEI/ASO complex from the injection site, and PEI/ASO-116 coated to MNBs versus PEI/ASO-116 alone were tested for their antitumoral effect in presence of a magnet implanted near the tumors. Consistent with the inhibitory effect on cell proliferation illustrated in Figure $5 \mathrm{C}$, tumor growth curves revealed a robust decrease in growth rates when treated with ASO-116 independent of the mode of delivery (Figure 5E). However, with increasing treatment tumors injected with ASO-116 under magnetic force-guidance were significantly smaller than those treated with PEI/ASO-116 alone. This demonstrates that by keeping the ASO concentrated in the tumor, we could enhance its specific therapeutic efficacy. Quantification of p73 transcripts in the tumors after final injection revealed an equally strong suppression of $\Delta \mathrm{Ex} 2 / 3 \mathrm{p} 73$ for the PEIand MNB/PEI-delivered antisense oligonucleotide (8.5fold and 7-fold) compared to the control groups (Figure $5 \mathrm{~F}$ ). Of note, enforced antitumorigenic activity of ASO116 directly correlated with the upregulation of the tumor suppressive TAp73 form, which showed a more than 2 times higher increase when tumors were subjected to magnetic force-guided ASO-116 treatment. These data suggest that inhibition of TAp73 in response to increased $\Delta \mathrm{Ex} 2 / 3 \mathrm{p} 73$ expression in growing tumors is the mechanism responsible for $\Delta \mathrm{Ex} 2 / 3 \mathrm{p} 73$-mediated tumor growth.
Based on present studies, NH2-truncated p73 isoforms are upregulated in the majority of tumors with a concomitant rise of TAp73 [9], suggesting that the specific ratio between the apoptotic TAp73 form and different dominant-negative 73 variants determines the functional outcome of p73 and the cell fate. In fact, alterations in the relative levels of truncated p73 and TAp73 and/or p53 correlate with therapy failure and poor prognosis in many cancers $[10,25,26]$. In this regard, downregulation of the $\Delta \mathrm{Np} 73$ species by antisense technique has been shown to alleviate its suppressive action and to enhance p53/ TAp73-mediated apoptosis in cancer cells in response to chemotherapy [3]. In turn, mice harbouring a specific knockout of TAp73 are tumor-prone and sensitive to carcinogens. Tomasini et al. reported that $73 \%$ of TAp $73 \%$ mice spontaneously develop malignancies, thereby establishing TAp73 isoforms as bona fide tumor suppressors [27]. Furthermore, this group showed that TAp73 is regulating the spindle assembly checkpoint (SAC) complex, suggesting that SAC impairment leads to genomic instability and aneuploidity in TAp73-deficient cells. Hence, the ability to induce and maintain proper mitotic arrest could be a mechanism of TAp73-mediated anti-tumorigenicity [28]. Both studies emphasize the functional importance of an imbalanced TAp73:DNp73 ratio. Taking into account that the expression levels of individual DNp73 species might be tumor-specific and particularly alternative-spliced isoforms $\Delta \mathrm{Ex} 2 / 3, \Delta \mathrm{Ex} 2$, and $\Delta \mathrm{N}^{\prime}$ that mimic the TAp73 knockout are predominantly expressed in many cancers including malignant melanoma $[10,29$ $33]$ rather than $\Delta \mathrm{Np} 73$, specific inhibitors for these potential oncogenes are needed. In this study, we show that selective knockdown of a single p73 splice product leads to the inhibition of melanoma tumor growth, and at the same time to the induction of TAp73, underscoring that the ratio between TAp73 and $\Delta \mathrm{Ex} 2 / 3 \mathrm{p} 73$ accounts for its oncogenic activity. Consistent with the induction of apoptosis after $\Delta \mathrm{Np} 73$ knockdown, ASO-mediated suppression of $\triangle T A p 73$ isoforms was sufficient to abrogate apoptosis resistance to chemotherapy mediated by ectopically expressed $\Delta T A p 73$. Enhanced chemosensitivity by inhibition of $\triangle T A p 73$ spliced isoforms was recently demonstrated in neuroblastoma using the cyclooxygenase inhibitor celecoxib, which blocks both $\Delta \mathrm{Ex} 2 / 3 \mathrm{p} 73$ and $\Delta$ Ex2p73 without isoform selectivity [34].

One of the most required desirements for LNA-ASOs is their efficient delivery to target cell nuclei. Polymers of cationic polyethyleneimine are well-studied compounds that improve the in vitro and in vivo penetrance of ASOs to cells and tissues [35], yet PEI-mediated DNA transfer is not tumor directed. Thus, we aimed at constructing targeted non viral vectors based on magnetic force-guided polyplexes by coating PEI/ASO to nanoparticles that allow 
A
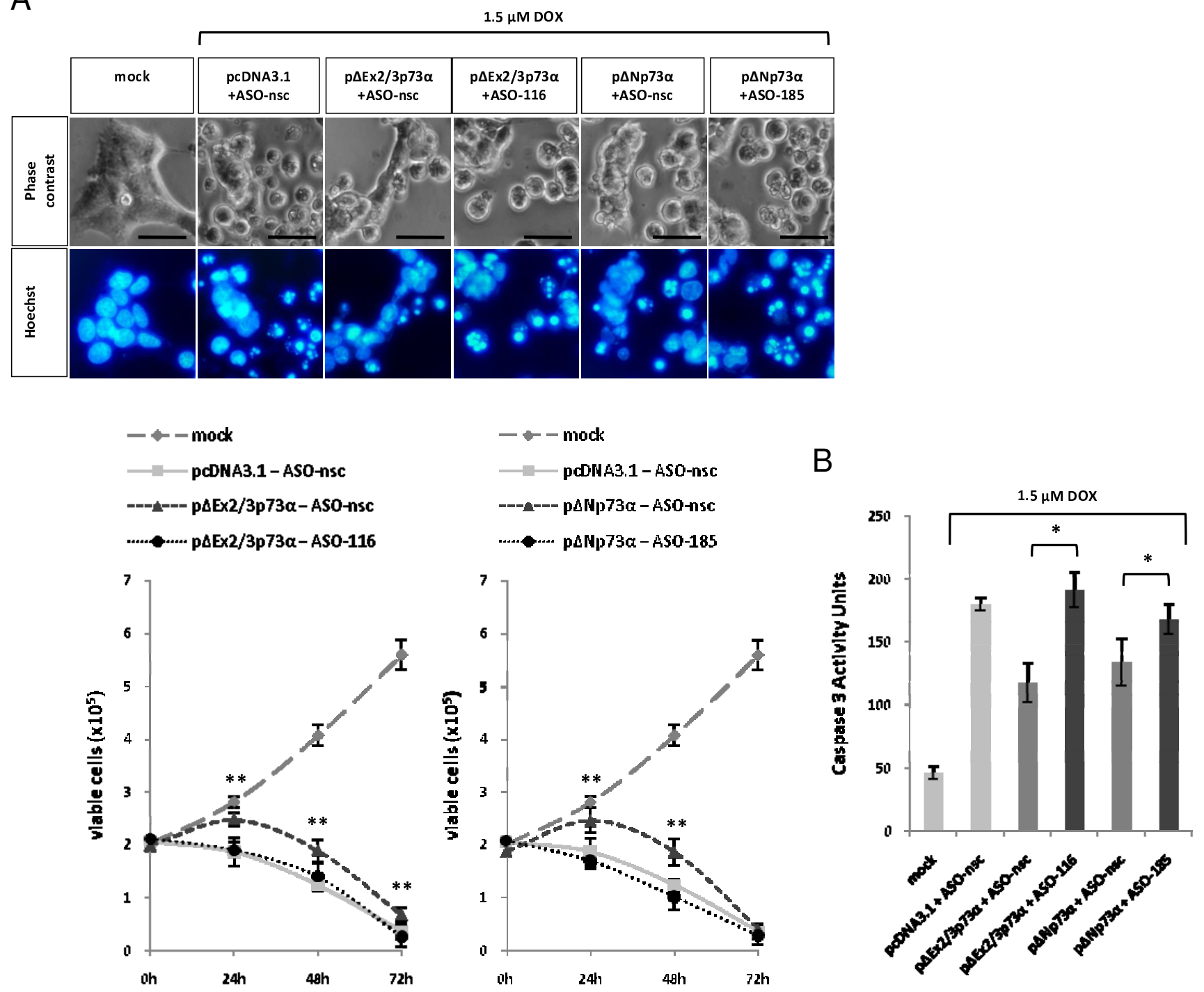

Figure 4

Induction of apoptosis in WI-38 fibroblasts by ASO-specific inhibition of DNp73. (A) Cells cultured in medium containing I.5 $\mu$ M doxorubicin (DOX) were stained with Hoechst 33342 at 48 hours after cotransfection. Apoptotic cells show the typical features of membrane blebbing, cell shrinkage, and nuclear condensation (upper panel). The amount of viable cells cotransfected with $\Delta \mathrm{Ex} 2 / 3 \mathrm{p} 73 \alpha$ or $\Delta \mathrm{Np} 73 \alpha$ in the absence and presence of specific ASO was counted at indicated time points after trypan blue exclusion. Black bars correspond to $50 \mu \mathrm{m}$. (B) Detection of caspase- 3 activity of cells treated as in A. Bar graphs show the mean \pm S.D. of three independent experiments. Asterisks denote statistical significant $p$-values: $*(p<0.05)$; ** $(p<0.005)$.

enforced cellular uptake by complex concentration at the tumor site. As a result, mice that received accumulating intratumoral injections of MNB/PEI-ASO complex against $\Delta \mathrm{Ex} 2 / 3$ showed a stronger degree of tumor growth inhibition than those treated only with PEI/ASO, indicating that increased antisense activity, substantiated by a marked induction of TAp73, correlates with higher transfection efficiency in tumor cells. Since the release of p73/p53 tumor suppressor function from $\triangle \mathrm{TAp} 73$-mediated inhi- bition has been linked to chemosensitivity [36], the use of oncogenic p73 ASOs as a therapeutic strategy for cancer treatment could be further improved by combination with other genotoxic agents.

\section{Conclusion}

We have developed LNA-modified ASOs that downregulate individual aberrantly expressed $\Delta \mathrm{Ex} 2, \Delta \mathrm{Ex} 2 / 3, \Delta \mathrm{N}^{\prime}$, and $\Delta \mathrm{N}$ p73 forms in neoplastic cells and growing tumors 
A

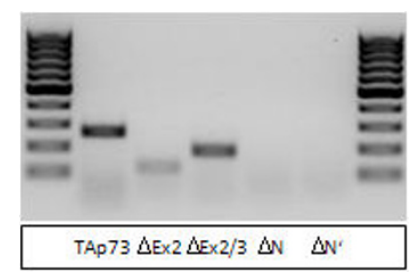

B

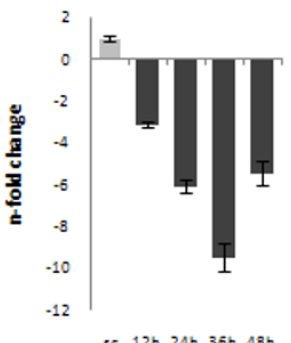

C

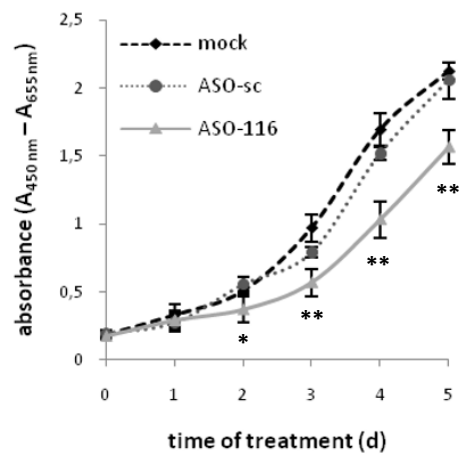

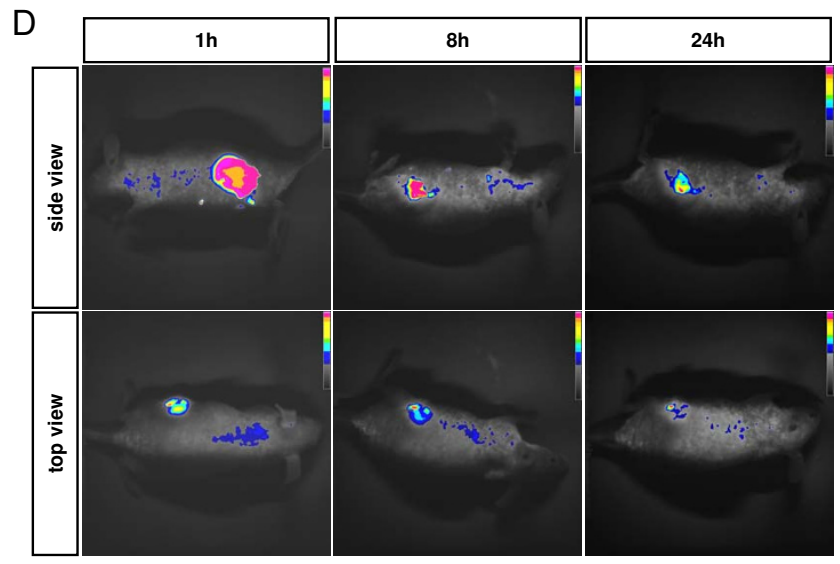

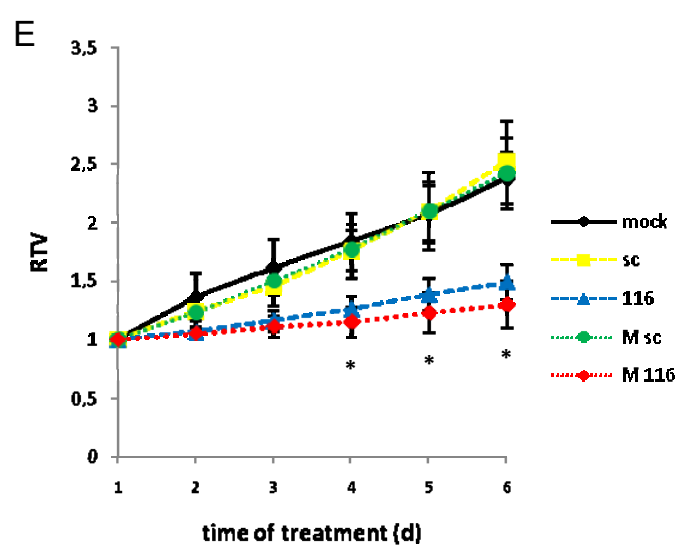

$\mathrm{F}$

In $\mathbf{n}=116$

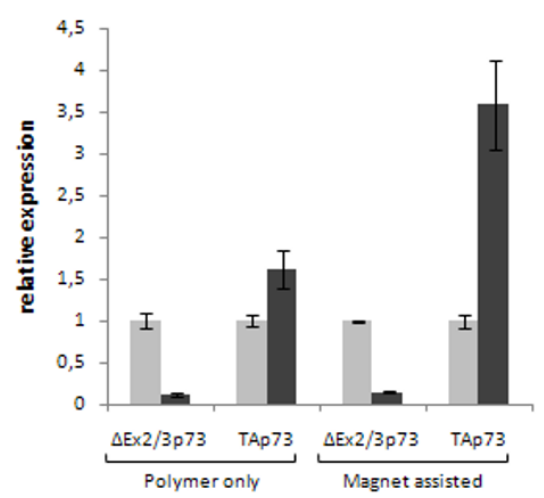

\section{Figure 5}

Activity of PEI/ASO-II6 in SK-Mel-29 cells and effect on tumor xenografts in vivo. (A) Semiquantitative RT-PCR analysis of endogenous p73 isoforms in melanoma cells. (B) Quantification of $\Delta$ Ex2/3p73 transcript levels in melanoma cells transfected with $250 \mathrm{nM}$ PEI/ASO-I 16 by real-time PCR at indicated time points. Expression was normalized to RPS9. Fold changes are relative to ASO-nsc. Data represent the mean \pm S.D. of three independent experiments. (C) BrdU incorporation of cells transfected with PEI/ASO-I I6- or ASO-sc on day I and 3 was measured by ELISA. Significant differences are labeled with asterisks $(* p<0.01 ; * * p<0.005)$. (D) In vivo imaging of PEI/ASO complex I, 8, and $24 \mathrm{~h}$ after intratumoral injection. The fluorescence image (pseudocolor) was overlaid on the photographic image. Intensity of fluorescent signal from Oregon green 488 labeled ASO is shown in top and side view. (E) Relative tumor volumes (RTV) of SK-Mel-29 xenografts injected with PEI/ ASO (sc and II6) or MNB/PEI/ASO (M sc and M II6) at a daily interval $(p<0.001$ ). Significant differences between M II6 and 116 are labeled with asterisks $(* p<0.05)$. (F) $\triangle E \times 2 / 3 p 73$ and TAp73 transcript levels in tumor tissues after 6 day treatment shown in E were quantitated by GRT-PCR. Expression was normalized to RPS9. Fold changes are relative to scrambled controls (set as I). Bars indicate mean values \pm S.D. of $n=4$ for each treatment. 
with high specificity. ASO-mediated modulation of endogenous isoform expression resulted in tumor growth inhibition in vivo accompanied by the induction of apoptotic TAp73. The data of this study support the application of NH2-truncated p73 inhibitors as valuable tools to delineate their biological role in human cancers and as anticancer agents.

\section{Competing interests}

The authors declare that they have no competing interests.

\section{Authors' contributions}

SE and BMP designed the study and wrote the manuscript. $\mathrm{SE}, \mathrm{KJ}$ and WW performed molecular studies. WL provided purified polymers. SE performed statistical analysis and data interpretation. All authors read and approved the final manuscript.

\section{Additional material}

\section{Additional file 1}

Design of DNp73-ASOs. Probability blots are shown as overlays of four $C$-terminal isoform constellations. The respective ASO target position is illustrated by the blue-shaded dashed line in each plot. $\triangle E x 2 p 73$ (A), $\triangle E x 2 / 3 p 73(B), \triangle N p 73(C)$, and $\triangle N^{\prime} p 73(D)$. (E-H) Secondary structure models indicate the ASO epitopes in the corresponding target transcripts, specific splice junctions in the epitopes of p $73 \Delta E \times 2$ and $\triangle E \times 2 / 3$ are labelled by double arrows. Predicted free energies $\Delta G$ are $917,0 \mathrm{kcal} / \mathrm{mol}$ for $\Delta E x 2 p 73(E), 866,3 \mathrm{kcal} / \mathrm{mol}$ for $\Delta E x 2 / 3 p 73(F), 901,2 \mathrm{kcal} / \mathrm{mol}$ for $\triangle \mathrm{Np} 73(\mathrm{G})$, and $1056,8 \mathrm{kcal} / \mathrm{mol}$ for $\triangle \mathrm{N}^{\prime} \mathrm{p} 73(\mathrm{H})$.

Click here for file

[http://www.biomedcentral.com/content/supplementary/14764598-8-61-S1.ppt]

\section{Additional file 2}

In silico calculated ASO binding energies. The data provided represent the binding energies of antisense oligonucleotides for each $\mathrm{N}$-terminal $p 73$ transcript with different C-terminal configurations.

Click here for file

[http://www.biomedcentral.com/content/supplementary/14764598-8-61-S2.ppt]

\section{Additional file 3}

Specific knockdown effect of ASO-115 and ASO-116 on DNp73 mRNA levels in tumor cells. H1299 cells with endogenously upregulated amino-truncated $p 73$ transcripts were treated with ASOs as shown in Figure 2. Fold expression was calculated after normalization with RPS9 relative to non-specific control-ASO (nsc). Bars indicate the mean \pm S.D. of three independent experiments.

Click here for file

[http://www.biomedcentral.com/content/supplementary/14764598-8-61-S3.ppt]

\section{Acknowledgements}

This work was supported by the Medical Faculty FORUN program grant 889012 and SFB Transregio 37. We thank Anja Stoll for technical assistance and Kathrin Sievert-Küchenmeister for giving advice in carrying out magnet-guided mice experiments.

\section{References}

I. Melino G, De Laurenzi V, Vousden KH: p73: Friend or foe in tumorigenesis. Nat Rev Cancer 2002, 2:605-6I5.

2. Stiewe T, Theseling CC, Putzer BM: Transactivation-deficient Delta TA-p73 inhibits $\mathrm{p} 53$ by direct competition for DNA binding: implications for tumorigenesis. J Biol Chem 2002, 277: $14177-14185$.

3. Zaika Al, Slade N, Erster SH, Sansome C, Joseph TW, Pearl M, Chalas E, Moll UM: DeltaNp73, a dominant-negative inhibitor of wildtype $\mathbf{p} 53$ and TAp73, is up-regulated in human tumors. J Exp Med 2002, 196:765-780.

4. Stiewe T, Stanelle J, Theseling CC, Pollmeier B, Beitzinger M, Putzer BM: Inactivation of retinoblastoma (RB) tumor suppressor by oncogenic isoforms of the p53 family member p73. J Biol Chem 2003, 278: 14230-14236.

5. Cam H, Griesmann H, Beitzinger M, Hofmann L, Beinoraviciote-Kellner R, Sauer M, Huttinger-Kirchhof N, Oswald C, Friedl P, Gattenlohner $S$, et al.: p53 family members in myogenic differentiation and rhabdomyosarcoma development. Cancer Cell 2006, 10:28I-293.

6. Petrenko O, Zaika A, Moll UM: deltaNp73 facilitates cell immortalization and cooperates with oncogenic Ras in cellular transformation in vivo. Mol Cell Biol 2003, 23:5540-5555.

7. Stiewe T, Zimmermann S, Frilling A, Esche H, Putzer BM: Transactivation-deficient DeltaTA-p73 acts as an oncogene. Cancer Res 2002, 62:3598-3602.

8. Tannapfel A, John K, Mise N, Schmidt A, Buhlmann S, Ibrahim SM, Putzer BM: Autonomous growth and hepatocarcinogenesis in transgenic mice expressing the p53 family inhibitor DNp73. Carcinogenesis 2008, 29:21।-218.

9. Buhlmann S, Putzer BM: DNp73 a matter of cancer: mechanisms and clinical implications. Biochim Biophys Acta 2008, 1785:207-216.

10. Dominguez G, Garcia JM, Pena C, Silva J, Garcia V, Martinez L, Maximiano C, Gomez ME, Rivera JA, Garcia-Andrade C, Bonilla F: DeltaTAp73 upregulation correlates with poor prognosis in human tumors: putative in vivo network involving p73 isoforms, p53, and E2F-I. J Clin Oncol 2006, 24:805-8I5.

II. Buhlmann S, Racek T, Schwarz A, Schaefer S, Putzer BM: Molecular mechanism of p73-mediated regulation of hepatitis $B$ virus core promoter/enhancer II: implications for hepatocarcinogenesis. J Mol Biol 2008, 378:20-30.

12. Fluiter $K$, ten Asbroek AL, de Wissel MB, Jakobs ME, Wissenbach M, Olsson $\mathrm{H}$, Olsen $\mathrm{O}$, Oerum $\mathrm{H}$, Baas $\mathrm{F}$ : In vivo tumor growth inhibition and biodistribution studies of locked nucleic acid (LNA) antisense oligonucleotides. Nucleic Acids Res 2003, 31:953-962.

13. Kurreck J, Wyszko E, Gillen C, Erdmann VA: Design of antisense oligonucleotides stabilized by locked nucleic acids. Nucleic Acids Res 2002, 30:1911-1918.

14. Crinelli R, Bianchi M, Gentilini L, Magnani M: Design and characterization of decoy oligonucleotides containing locked nucleic acids. Nucleic Acids Res 2002, 30:2435-2443.

15. Ding $Y$, Lawrence CE: A bayesian statistical algorithm for RNA secondary structure prediction. Comput Chem 1999, 23:387-400.

16. Ding Y, Lawrence CE: Statistical prediction of single-stranded regions in RNA secondary structure and application to predicting effective antisense target sites and beyond. Nucleic Acids Res 2001, 29: 1034-1046.

17. Mathews DH, Turner DH: Prediction of RNA secondary structure by free energy minimization. Curr Opin Struct Biol 2006, 16:270-278.

18. Ding Y, Chan CY, Lawrence CE: Sfold web server for statistical folding and rational design of nucleic acids. Nucleic Acids Res 2004, 32: |35-|4|.

19. Scherr M, Rossi JJ, Sczakiel G, Patzel V: RNA accessibility prediction: a theoretical approach is consistent with experimental studies in cell extracts. Nucleic Acids Res 2000, 28:2455-246I.

20. Frank P, Albert S, Cazenave C, Toulme J]: Purification and characterization of human ribonuclease HII. Nucleic Acids Res 1994, 22:5247-5254. 
21. Reynolds A, Leake D, Boese Q, Scaringe S, Marshall WS, Khvorova A: Rational siRNA design for RNA interference. Nat Biotechnol 2004, 22:326-330.

22. Grob TJ, Novak U, Maisse C, Barcaroli D, Luthi AU, Pirnia F, Hugli B, Graber HU, De Laurenzi V, Fey MF, et al:: Human delta Np73 regulates a dominant negative feedback loop for TAp73 and p53. Cell Death Differ 200I, 8: I 2I3-I223.

23. Soengas MS, Capodieci P, Polsky D, Mora J, Esteller M, Opitz-Araya X, McCombie R, Herman JG, Gerald WL, Lazebnik YA, et al.: Inactivation of the apoptosis effector Apaf-I in malignant melanoma. Nature 200I, 409:207-2II.

24. Li W, Ma N, Ong LL, Kaminski A, Skrabal C, Ugurlucan M, Lorenz P, Gatzen HH, Lutzow K, Lendlein A, et al:: Enhanced thoracic gene delivery by magnetic nanobead-mediated vector. J Gene Med 2008, I 0:897-909.

25. Casciano I, Mazzocco K, Boni L, Pagnan G, Banelli B, Allemanni G, Ponzoni M, Tonini GP, Romani M: Expression of DeltaNp73 is a molecular marker for adverse outcome in neuroblastoma patients. Cell Death Differ 2002, 9:246-25I.

26. Muller M, Schilling T, Sayan AE, Kairat A, Lorenz K, Schulze-Bergkamen H, Oren M, Koch A, Tannapfel A, Stremmel W, et al:: TAp73/ Delta Np73 influences apoptotic response, chemosensitivity and prognosis in hepatocellular carcinoma. Cell Death Differ 2005, I 2: I564-I577.

27. Tomasini R, Tsuchihara K, Wilhelm M, Fujitani M, Rufini A, Cheung CC, Khan F, Itie Youten A, Wakeham A, Tsao MS, et al.: TAp73 knockout shows genomic instability with infertility and tumor suppressor functions. Genes Dev 2008, 22:2677-269l.

28. Tomasini R, Tsuchihara K, Tsuda C, Lau SK, Wilhelm M, Ruffini A, Tsao MS, lovana JL, Jurisicova A, Melino G, et al.: TAp73 regulates the spindle assembly checkpoint by modulating BubR I activity. PNAS 2009, I06(3):797-802.

29. Tuve S, Wagner SN, Schittek B, Putzer BM: Alterations of DeltaTA-p73 splice transcripts during melanoma development and progression. Int J Cancer 2004, I08: I62-I66.

30. O'Nions J, Brooks LA, Sullivan A, Bell A, Dunne B, Rozycka M, Reddy A, Tidy JA, Evans D, Farrell PJ, et al.: p73 is over-expressed in vulval cancer principally as the Delta 2 isoform. Br J Cancer 200I, 85: $|55|-\mid 556$.

31. Stiewe T, Tuve S, Peter M, Tannapfel A, Elmaagacli AH, Pützer BM: Quantitative TP73 transcript analysis in hepatocellular carcinomas. Clin Cancer Res 2004, 10:626-633.

32. Wager M, Guilhot J, Blanc JL, Ferrand S, Milin S, Bataille B, Lapierre F, Denis S, Chantereau T, Larsen CJ, Karayan-Tapon L: Prognostic value of increase in transcript levels of Tp73 DeltaEx2-3 isoforms in low-grade glioma patients. $\mathrm{Br} J$ Cancer 2006, 95:1062-1069.

33. Castellino RC, De Bortoli M, Lin LL, Skapura DG, Rajan JA, Adesina AM, Perlaky L, Irwin MS, Kim JY: Overexpressed TP73 induces apoptosis in medulloblastoma. BMC Cancer 2007, 7:127.

34. Lau LM, Wolter JK, Lau JT, Cheng LS, Smith KM, Hansford LM, Zhang L, Baruchel S, Robinson F, Irwin MS: Cyclooxygenase inhibitors differentially modulate p73 isoforms in neuroblastoma. Oncogene 2009, 28:2024-2033.

35. Seong J-H, Lee K-M, Kim ST, Jin S-E, Kim C-K: Polyethyleniminebased antisense oligonucleotides of IL-4 suppress the production of IL-4 in a murine model of airway inflammation. J Gene Med 2006, 8:314-323.

36. Irwin MS, Kondo K, Marin MC, Cheng LS, Hahn WC, Kaelin WG Jr: Chemosensitivity linked to p73 function. Cancer Cell 2003, 3:403-410
Publish with Biomed Central and every scientist can read your work free of charge

"BioMed Central will be the most significant development for disseminating the results of biomedical research in our lifetime. "

Sir Paul Nurse, Cancer Research UK

Your research papers will be:

- available free of charge to the entire biomedical community

- peer reviewed and published immediately upon acceptance

- cited in PubMed and archived on PubMed Central

- yours - you keep the copyright

Submit your manuscript here:

http://www.biomedcentral.com/info/publishing_adv.asp
BiolMedcentral 